\author{
A.Y. Ovchinnikov ${ }^{1}$, Y.L. Soldatskiy ${ }^{2,3}$, A.M. Mityuk ${ }^{1}$ \\ ${ }^{1}$ Evdokimov Moscow State Dental Medical University, Russian Federation \\ ${ }^{2}$ Morozovskaya children's municipal clinical hospital, Moscow, Russian Federation \\ ${ }^{3}$ Pirogov Russian National Medical Research University, Moscow, Russian Federation
}

\title{
New view on cough treatment in children
}

\section{Author affiliation:}

Soldatskii Yurii L'vovich, $\mathrm{PhD}$, Professor, head of the otolaryngology service at the Morozovskaya children's municipal clinical hospital, professor of the otolaryngology department of the pediatrics faculty at the Pirogov RNMRU

Address: $1 / 9,4^{\text {th }}$ Dobryninskiy Ln., Moscow,119049, tel.: +7 (499) 236-24-02

Article received: 02.04.2013, accepted for publication:

The article is dedicated to cough treatment in children as one of the most frequent causes of references to pediatricians. The authors explain mechanisms and causes of this symptom - both frequent and rare. The latter often lead to underestimation of the situation, incorrect diagnostics and, therefore, to ineffective treatment. Primarily, such conditions involve cough at gastroduodenal reflux disease. The article presents a detailed review of drugs for cough treatment. It examines a phytogenic drug with one of the best efficacy and safety profiles in detail.

Keywords: cough, mechanism, causes, treatment, children.

Cough is the fifth most frequent cause of outpatient visits to doctors. It is the most frequent symptom caused by pathology of the respiratory system. Cough is a protective-adaptive reaction, which provides elimination of irritant endogenous (mucus, sputum, pus) or exogenous agents (foreign bodies, dust particles etc.) from the respiratory tract. In order to establish correct diagnosis and select adequate therapy it is necessary to identify the cause of cough. This requires appraisal of all characteristics of cough. Each of them has a certain diagnostic value. However, response of the parents as to how long has the child been having cough allows the doctor to assume its possible cause.

Acute cough. The most frequent causes of acute cough are such upper respiratory tract infections as acute respiratory viral infections (ARVI), acute bacterial sinusitis, pertussis, exacerbation of chronic obstructive pulmonary diseases, allergic rhinitis. The most frequent cause of acute cough is viral upper respiratory tract infections. Frequency of this symptom in the untreated ARVI patients reaches $83 \%$ within 48 hours of the disease and reduces down to $26 \%$ by day 14 . Cough at ARVI is stimulated by the nasopharyngeal discharge drip and irritation of pharyngeal mucous tunic. Cough passes completely in case ARVI is treated adequately. However, sometimes cough may become persistent.

Persistent cough. Usually, it starts with an upper respiratory tract infection and continues for 3-8 weeks; it usually is post-infectious or indicates bacterial sinusitis or asthma.

Causes of chronic cough are:

1. Postnasal drip syndrome of mucus into pharynx due to rhinitis or sinusitis. "Postnasal drip syndrome" (PNDS) is clinical situations characterized by inflammatory process of the upper respiratory tract (nasopharynx, nose, paranasal sinuses), when nasal discharge drips from the back wall of pharynx into the tracheobronchial tree. Differential diagnosis of the PNDS-induced cough includes bacterial sinusitis, post-infectious rhinitis, allergic rhinitis, drug-induced rhinitis, rhinitis gravidarum etc. In recent years it has been suggested to use the term "upper airway cough syndrome" (UACS) as cough may be caused not only be nasal and nasopharyngeal mucus drip, but also inflammation of the upper respiratory tract in the area of cough receptors [1]. Doctors' awareness of this syndrome is important, as cough in this situation is not always correctly interpreted and 
erroneously considered a symptom of chronic bronchitis. This syndrome is the most frequent cause of chronic cough occurring in 20-34\% of patients with chronic cough.

2. Gastroesophageal reflux disease (GERD) is one of the main causes of chronic cough. Gastroesophageal reflux is considered to be the cause of chronic cough in more than $20 \%$ of adult patients [2]. GERD causes cough somewhat rarer in children - less than $10 \%$ of cases [3]. Diagnosis of the GERD-associated chronic cough may be complicated due to the lack of typical reflux symptoms, such as heartburn and regurgitation, in many patients [4]. The GERD-associated chronic cough may be caused directly by aspiration of refluxate or due to the vagus-mediated esophageal tracheobronchial reflex when gastric material affects vagus receptors of the distal segment of esophagus $[5,6]$.

3. Specific variant of bronchial asthma called non-asthmatic cough, most often nocturnal. The so called cough bronchial asthma is present in $20-28 \%$ of all cases of chronic cough.

Causes of cough in children are numerous [2-9]. Most often cough in children is a symptom of an infectious-inflammatory process in the upper and lower respiratory tracts. Cough in children is often caused by allergic inflammation of the respiratory tract's mucous tunics, bronchospasm and respiratory tract's obstruction with viscous bronchial secretion / aspirated foreign bodies. Persistent cough in children may be caused by passive smoking, abnormal psychoemotional state, postnasal drip syndrome. Perhaps, the most frequent cause of chronic cough in children is postnasal drip syndrome. Nasal secretion drip into pharynx and larynx manifests itself differently at different times of the day. At night, when a child sleeps horizontally, this mucus drips to laryngopharynx, irritates reflexogenic zones and causes cough. At day, when a child is in the vertical position, the mucus drips to laryngopharynx as well, but is swallowed on the reflex level, reaches epiglottis and vocal cords in little portions, thus, it does not cough. Postnasal drip syndrome manifests itself as the cause of persistent cough primarily at night. Usually, auscultation reveals vesicular respiration and no rales. In some patients cough may be the only reason of UACS. Clinical manifestations of the syndrome are non-specific, thus, its ultimate identification on the basis of anamnestic and physical examination data only is impossible. Connection of chronic cough to pathology of the upper respiratory tract is not always clearly tractable and thus requires additional confirmation.

Along with hoarseness, cough is the one of the primary symptoms of acute and chronic laryngitis. Laryngitis is characterized by dry hoarse whooping cough. Acute laryngitis in children is caused by a respiratory viral infection in a vast majority of cases. Chronic laryngitis may develop due to numerous exogenous and endogenous factors, such as unfavorable environmental conditions, high vocal stress, diseases of other organs and systems (primarily, of the gastrointestinal tract) etc. It should be noted that persistent cough, e.g. psychogenic persistent cough may directly cause damage to mucous tunic of vocal cords and development of chronic laryngitis. Moreover, various neoplastic pharyngeal process (the most frequently occurring in childhood being recurrent respiratory papillomatosis may be accompanied by cough, especially at ballottement of neoplasm in the fissure of glottis [7]. According to Clement (2006), the most frequent cause of cough in children is viral upper respiratory tract infections, their hyperreactivity, GERD and other causes. Although cough may be associated with sneezing and respiratory tract's dysfunction, it should be remembered that chronic cough might only manifest respiratory tract's hyperreactivity.

Cough may develop due to high concentration of harmful airborne substances (gas contamination, tobacco smoke), extremely dry and overheated air in the room. Cough may also be caused by the diseases that are not connected with respiratory system. E.g., it may develop in children with heart diseases or gastrointestinal tract's pathology. One of the rarer causes is psychogenic (reflex) cough, which occurs, e.g., in case of pathology of the external acoustic meatus (cerumen impaction) and the middle ear (in case of its inflammation, regardless whether it is acute or chronic).

There are other, rarer causes of chronic cough. Chlamidia trachomatis pneumonia may be suspected in children of 1-3 months of age with cough, tachypnea and conjunctivitis. 
Tuberculosis and fungal infections may cause dry, hoarse cough due to compression or expansion of lymph nodes. Pertussis may start with rhinorrhea and soft cough progressing to paroxysmal. Diseases involving the system of the respiratory tract's immune protection are represented by recurrent pneumonias and constant respiratory symptoms. Frequent respiratory infections reoccurring after the course of antibacterial therapy are the first manifestation of the immune system's failures. They may lead to the development of bronchiectases. Lymphocytic interstitial pneumonia as complication of HIV-infection manifests itself clinically with nonproductive chronic cough and respiratory dysfunction.

In infants, cough may be connected with microaspiration of gastric material and saliva; parents may deny their child's posseting and regurgitation. Usually, microaspiration is causes by the increased intra-abdominal pressure in children with abnormal laryngeal development, primarily by laryngomalacia. The primary symptom of this anomaly is inspiratory stridor, which may be stable or aggravate during feeding or when the child is anxious. Aspiration syndrome may be caused by other laryngeal structure anomalies as well - laryngeal tracheoesophageal cleft, isolated tracheoesophageal fistula, agenesis of epiglottis etc.; diagnostics of these diseases requires detailed endoscopic examination of the respiratory tract [10]. The most frequent cause of aspiration syndrome in children under 1 year of age is neurological pathology.

Other lesions, from pulmonary sequestration to bronchogenic cysts may be asymptomatic or result in stable, persistent cough due to a respiratory tract infection or compression. Mediastinal tumors may cause cough, dysfunction of vocal cords or superior vena cava syndrome. Chronic cough may also cause acyanotic congenital heart diseases (defects of ventricular septum, unclosed arterial duct, pulmonary stenosis, Fallot's tetrad) due to bronchial compression with increased pulmonary arterial pressure, expansion of the left atrium or constriction of the peripheral respiratory tract due to pulmonary edema.

Sudden cough in children may signal aspiration of a foreign body; this poses severe threat to the child's life and requires immediate intervention of a doctor. Aspiration of a foreign body has a bright clinical presentation: abrupt paroxysmal cough in the setting of full health, possibly, asphyxia with loss of consciousness and cyanosis with subsequent stenotic respiration and frequently recurring episodes of pertussis-like cough; however, aspiration may not be manifested with many symptoms in infants due to underdeveloped reflexogenic laryngeal zones. Foreign body in lower segments of the respiratory tract may cause emphysema, atelectasis, recurrent pneumonias, although it may be present for a long time without many symptoms. Movement of the foreign body may cause paroxysmal cough accompanied by cyanotic episodes, which may be as bad as asphyxia. Tracheobronchoscopy helps to clarify diagnosis.

Despite a seeming variety of causes of cough, its cause may be correctly diagnosed in $88-100 \%$ of patients; successful treatment may be conducted in $84-98 \%$ of them. The key to success in this situation is to establish correct diagnosis and eliminate cause of cough.

When treating cough, it is necessary to take into consideration its etiology, treatment and character. Etiotropic therapy, which presupposes either elimination of the cause of cough (elimination of contact with an allergen, removal of cerumen impaction from the external acoustic meatus etc.) or liquidation of the cough-inducing pathological process (antibacterial therapy of pneumonia and bacterial rhinosinusitis, treatment of gastroesophageal reflux etc.) is the most effective. In other cases, when etiologic or pathogenetic therapy is somehow impossible or insufficiently effective, cough is treated with symptomatic therapy. Selection of drugs in that case depends primarily on the character of cough, its intensity and other peculiarities.

Antitussive drugs are divided by action mechanism into:

- Central narcotic (codeine) and non-narcotic (glaucin) drugs, which cause inhibition of the cough center;

- Peripheral drugs, which reduce sensitivity of cough receptors (local anesthetics, multipurpose drugs and the drugs suppressing release of neuroleptics). 
Formation of viscous hardly sequestrable sputum is an important pathogenetic mechanism of the bronchial conductivity disorder. Dilution and removal of this secretion has an important place in cough treatment. The drugs affecting sputum formation may be divided into 4 groups $[6,7,9]$ :

- Reflex action drugs, which realize their effect using gastric mucous tunic (thermopsis) and chemoreceptor trigger zone (apomorphine). They irritate afferent fibers of the parasympathetic nerve and gastric mucous tunic; this stimulates the respiratory center on the reflex level. It results in the stimulation of bronchial glands, higher mucus secretion, higher peristalsis of bronchioles and higher activity of bronchial ciliated epithelium; in its turn, this promotes sputum evacuation. It should be noted that bronchial congestion with the excessive amount of sputum may occur in smaller children, which they are unable to expectorate.

- Drugs affecting mucous tunic of the respiratory tract directly (saline, alkalis, essential oils). Iodides are the most popular among them. Discharging from blood, iodine salts cause hyperemia of bronchial mucous tunic and stimulate bronchial glands, thus, the sollayer becomes more fluid.

- Proteolytic enzymes.

- Stimulators of surfactant products (ambroxol and its derivatives).

Drugs inducing cough and sputum discharge are used in case of non-productive cough in debilitated patients.

Despite the variety of antitussive drugs, the search for new, effective and safe drugs remain relevant. Medicinal plants, such as primrose flowers, sorrel herbs, elder flowers, vervain herbs, gentian roots have long been used in medicine to treat respiratory tract diseases. Sinupret (Bionorica, Germany), which was developed more than 60 years ago and has a long history of practical application, includes these very medicinal plants. The drug is characterized by the unique complex selection of the combination of medicinal plants. It was created using a special manufacturing technology - phytoneering, which allows reaching the maximal concentration of active materials at minimal allergenicity, good tolerability and high safety profile. The fivecomponent composition allows the phytogenic drug to have secretolytic, anti-inflammatory, antiviral and immunomodulating action, which has been proven by clinical trials [11-14].

Sinupret effectively regulates secretion and normalizes mucus viscosity, eliminates mucostasis and recovers mucociliary clearance. Decrease in viscosity of the secretion occurs due to the stimulation of secretory cells of the upper respiratory tract's mucous tunic, which produce neutral mucopolysaccharides. The drug has anti-inflammatory and anti-edematous effect on the mucous tunic suppressing synthesis of pro-inflammatory mediators [12] and reducing edema in the area of fistulas of paranasal sinuses. The conducted trials [15] revealed increase in activity of the non-specific protection factors and reinforcement of local immunity of mucous tunics in the setting of its application: significant increase in the amount of phagocytes, saliva levels of sIgA and lysozyme, increase in the concentration of immunoglobulins $\mathrm{M}$ and $\mathrm{A}$ in blood serum. Increase in activity of the antiviral immunity due to increase in the production of a combination of interferon $\alpha$ and interferon $\gamma$ has been revealed at the same time. These properties of the drug considerably reduce the risk of postnasal drip syndrome developing due to acute and chronic rhinosinusites of both viral and bacterial nature and the cough they induce. The drug activates local and common factors of immune protection and reduces duration of acute respiratory diseases. Immunomodulating action has been confirmed by clinical and laboratory data of the trial conducted by G.I. Smirnova (2012). It should also be noted that Sinupret may be used to treat and prevent acute and chronic rhinosinusitis in all patients, including children over 2 years of age.

In her trial, Professor T.A. Mashkova of the Burdenko Voronezh State Medical Academy assessed results of treatment of 52 children with ARVI [15]. All patients complained of indisposition, mild pyrexia, disturbed nasal breathing as bad as the complete nasal congestion in most patients $(64.3 \%)$ and voluminous nasal discharge $(81 \%)$ at the primary visit. At the first endoscopic examination, otolaryngologists revealed pronounced phenomena of acute 
rhinopharyngitis, which was complicated with cough in $58 \%$ of cases. The first group of patients was prescribed monotherapy with Sinupret, the referent group - endonasal irrigation therapy, decongestants and antiseptics. Observation results of Professor T.A. Mashkova showed improvement of the general condition, including cough decrease, by day 3 in $64.5 \%$ of patients, by day $4-5$ - in $19.4 \%$, by day $7-8$ - in $14.3 \%$. Cough terminated completely by day 7 of treatment in most patients of the first group. Comparative observation analysis showed improvement of the general condition by day 5-6 (47.9\% of the referent group patients), recovery (including termination of cough) by day 10-11; condition improved by day 7-8 in $52.1 \%$ of patients, recovery occurred only by day $13-14$.

Thus, the given review of treatment results of children with ARVI showed that the phytopreparations promotes milder course of the disease. Thanks to anti-inflammatory, antiedematous, secretolytic and antiviral effect, the drug prevents development of complications and accelerates recovery processes by $45 \%$ in tote, reduces the disease duration and cough occurring due to viral rhinitis. This allows us to conclude that Sinupret has a rather high therapeutic efficacy in terms of termination of the cough induced by postnasal drip syndrome. It should also be noted that the drug is well tolerated and lacks undesirable phenomena.

\section{REFERENCES}

1. Pratter M. R. Chronic upper airway cough syndrome secondary to rhinosinus diseases (previously referred to as postnasal drip syndrome): ACCP evidence-based clinical practice guidelines. Chest. 2006 Jan; 129 (1 Suppl.): 63S-71S.

2. Kastelik J. A., Aziz I., Ojoo J. C., Thompson R. H., Redington A. E., Morice A. H. Investigation and management of chronic cough using a probability-based algorithm. Eur Respir J. 2005 Feb; 25 (2): 235-43.

3. Marchant J. M., Masters I. B., Taylor S. M., Cox N. C., Seymour G. J., Chang A. B. Evaluation and outcome of young children with chronic cough. Chest. 2006 May; 129 (5): 113241.

4. Saritas Yuksel E., Vaezi M. F. New developments in extraesophageal reflux disease. Gastroenterol Hepatol (N. Y.). 2012 Sep; 8 (9): 590-9.

5. Vakil N., van Zanten S. V., Kahrilas P., Dent J., Jones R. Global Consensus Group. The Montreal definition and classification of gastroesophageal reflux disease: a global evidencebased consensus. Am J Gastroenterol. 2006 Aug; 101 (8): 1900-20.

6. Namazova-Baranova L. S., Nisevich L. L., Volkov K. S., Vazhnova I. M. Pediatricheskaya farmakologiya - Pediatric pharmacology. 2008; 5 (3): 64-71.

7. Soldatskii Yu. L. Pediatricheskaya farmakologiya - Pediatric pharmacology. 2008; 5 (3): 68.

8. K. Volkov, L. Namazova, L. Nisevich. Clamydia, mycoplasma and viral infections in children with chronic cough. Abstracts for the 3rd Europaediatrics Congress. 2008. P. 400.

9. Volkov K. S., Nisevich L. L., Namazova-Baranova L. S., Filyanskaya E. G., Alekseeva A. A., Barannik V. A. Voprosy sovremennoi pediatrii - Current pediatrics. 2013; 12 (1): 112-116. 10. Striga E. V., Zaitseva O. V., Soldatskii Yu. L., Onufrieva E. K. Pediatriya - Pediatrics. 2010; 89 (2): 86-90.

11. Ryazantsev S. V., Zakharova G. P., Drozdova M. V. Novosti otolaringologii i logopatologii - News of otolaryngology and logopatologii. 2002. pp. 102-105.

12. Springer Medizin. Urben@Vogel Gmbh Munchen. September, 2011.

13. Glatthaar-Saalmuller B., Rauchhaus U., Rode S., Haunschild J., Saalmuller A. Antiviral activity in vitro of two preparations of the herbal medicinal product Sinupret against viruses causing respiratory infections. Copyright, 2011. Elsevier GmbHPhytomedicine. 2011 Dec 15; 19 (1): $1-7$.

14. Ito H. et al. Immunological studies of host-mediated antitumor agents in sarcoma-180bearing mice. Jap Jour Pharm. 1982; 32: 225. 
15. Mashkova T. A., Mal'tsev A. B. Voprosy pediatrii - Problems of pediatrics. 2012; 2. 